Proceedings of the 2006 Winter Simulation Conference

L. F. Perrone, F. P. Wieland, J. Liu, B. G. Lawson, D. M. Nicol and R. M. Fujimoto, eds.

\title{
THE IMPACT OF ORDINAL ON RESPONSE SURFACE METHODOLOGY
}

\author{
Sara Jian Oon \\ Raffles Junior College \\ 10 Bishan Street 21, Singapore 574013
}

\author{
Loo Hay Lee \\ Department of Industrial and Systems Engineering \\ National University of Singapore \\ 10 Kent Ridge Crescent, Singapore 119260
}

\begin{abstract}
Traditionally, Response Surface Methodology (RSM) is cardinal in nature. Ordinal optimization was only introduced recently. Since ordinal optimization has been proven to be successful in certain applications, this paper aims to investigate whether ordinal optimization improves RSM by developing ordinal RSM and comparing it with cardinal RSM in terms of efficiency, accuracy and consistency. Assuming that the performances of systems can be expressed as functions of their parameters, both ordinal and cardinal RSM are simulated for several simple multivariable mathematical functions and the effectiveness of ordinal RSM evaluated. It was found that ordinal does not always improve RSM, especially in functions which exhibit a large gradient change over a small region.
\end{abstract}

\section{INTRODUCTION}

In this paper we investigate how ordinal optimization improves RSM. Ordinal optimization was proposed by Ho et al. (1992). It involves the use of the ranking of noisy performance estimates instead of the actual performance, which can be difficult to determine. Also, ordinal optimization aims to find a subset in which "good enough" designs are contained with high probability, instead of trying to obtain one true maximum, which will be difficult in the presence of large noise.

RSM, invented by Box and Wilson (1951), is a collection of mathematical and statistical techniques for empirical model building. By careful design of experiments, the objective is to optimize a response (output variable) which is influenced by several independent variables (input variables). An experiment is a series of tests, called runs, in which changes are made in the input variables in order to identify the reasons for changes in the output response (Montgomery \& Runger 1994). RSM involves two basic concepts:

(1) the choice of the approximate model, and

(2) the plan of experiments where the response has to be evaluated.
In combinatorially explosive stochastic optimization problems, RSM provides a major advantage by mapping the response surface over a particular region of interest. Hence, it is easier to select operating conditions to achieve desired specifications and optimize the response (Box 1987 and Myers 2002). This enables a designer to efficiently allocate resources for detailed analyses and improvements of these potential solutions.

Conventionally, cardinal optimization is used in RSM, where the actual performances are used in the evaluation of experimental responses. Due to the presence of large noise, the actual performances cannot be determined confidently, resulting in inaccuracy in subsequent steps. In ordinal optimization, the relative ranks of the responses are used instead. The relative orders of the responses can be determined with more accuracy; hence the desired region in which the optimum can be mapped more accurately and the latter obtained more efficiently. In fact, it has been investigated that ordinal comparison does exhibit much better convergence property than value estimation (Dai 1996, Xie 1997, and Dai and Chen 1997,Lee et. al. 1999). Moreover, accurate performances take a long time to obtain, whereas the relative order of performance estimates can be determined within a shorter time.

Ordinal optimization has been applied successfully to many situations, such as the 10-node network (Patsis et al. 1997), rare event simulations (Ho and Larson 1995) and apparel manufacturing system (Lee et. al. 2000). Hence, we have decided to incorporate ordinal optimization into RSM to see if RSM can be improved by this modification.

While it has been widely argued and acknowledged that ordinal optimization is preferred to cardinal optimization, this paper concentrates on integrating the concept of ordinal optimization in the RSM. We call this new method Ordinal RSM . We compare the two RSMs based on efficiency, accuracy and consistency as these are the key factors that designers are concerned with, primarily because finding a good design in a short time will lead to better resource allocation.

In Section 2, we will discuss the general RSM and the development of the ordinal RSM. In Section 3, we will 
demonstrate how to implement the RSM experiments, and the numerical results will be summarized in Section 4. Finally Section 5 concludes the findings.

\section{RESPONSE SURFACE METHODOLOGY}

\subsection{Cardinal RSM}

In any complex stochastic system, the performance can conceptually be given by $J(\theta)$ where $J$ is the expected performance measure and $\theta$ are the system design parameters which may be continuous, discrete, combinatorial or even symbolic. A general problem of stochastic optimization can be defined as:

$$
\min _{\theta \in \Theta} J(\theta) \equiv E[L(\theta, \xi)],
$$

where $\Theta$, the search space, is an arbitrary, huge, structureless but finite set; $\theta$ is the system design parameter vector; $J$ is the performance criterion which is the expectation of $L$, the sample performance, as a function of $\theta$ and $\xi$, a random vector that represents uncertain factors in the system .

The first step of RSM is to find a suitable approximation for the function $L$. The most common forms are loworder polynomials but can be generalized with the inclusion of any mathematical operator (e.g. trigonometric functions). Once an approximate model is obtained, the goodness-of-fit determines if the solution is satisfactory. If this is not the case, the process is restarted and further experiments are made. In this paper, we do not conduct physical experiments, as the focus lies more in the second step; therefore an assumed function is used to carry out the second step.

In the second step, the function is modeled mathematically in an attempt to hunt for the optimum response. The method of steepest ascent (or descent) can be used. It is a procedure for moving sequentially along the path of steepest ascent, in the direction of the maximum increase in the response. This is based on the assumption that within a small region or in a region far from the optimum, a firstdegree model can be approximated (Montgomery \& Runger 1994). For a relatively small region of the response surface, the contours of the function can be taken to be parallel and hence the direction of steepest ascent would be directly perpendicular to the contours. Experiments are conducted on this path until the response no longer increases. At every point, a linear regression analysis is performed on the data, and the gradient is calculated, as the step size has to be proportional to the regression coefficient.

For this stage, we have chosen to adopt a modified version of the central composite design (CCD). CCDs traditionally involve 1 central point, $2^{\mathrm{N}}$ factorial points and $2 \mathrm{~N}$ axial points, where $\mathrm{N}$ is the number of parameters. In our experiments, the axial points have been eliminated as the step sizes are relatively small; hence the factorial points are sufficient to maintain an adequate level of accuracy.

Another limitation is the presence of local maxima in some systems. This has to be overcome by restarting using different input values to see whether the same optimum is reached. However, this is not the focus of this paper and will not be addressed in the subsequent sections. Our aim is to compare how close the values obtained by the two methods are to an optimum, especially if they locate one of the same height, and also the number of steps needed to reach the point close of the optimum. The question of local and global optimums can be addressed in future studies.

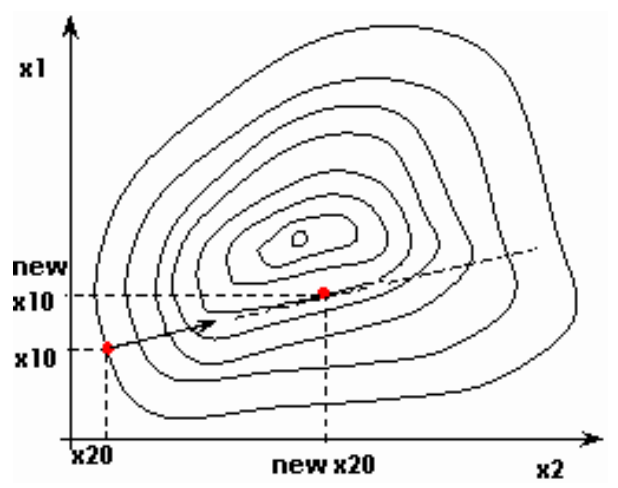

Figure 1: Contours of a Response Surface for Which RSM is Used to Locate the Maximum.

\section{Outline of the Typical RSM}

Step 1: Letting $n=0$, the initial central point is denoted by $x_{0}$ where $x_{0}$ is a vector

Step 2: Let the $\delta x_{n}$ be the chosen distance between the central and factorial points, such that the factorial points are denoted by $x_{n} \pm \delta x_{n}$.

Step 3: Evaluate the responses on all the design points.

Step 4: Calculate the gradient, $\Delta F_{n}$, of the response surface in the vicinity of the central point.

Step 5: Finding the next central value, $x_{n+1}$,

$$
x_{n+1}=x_{n} \pm \frac{\Delta F_{n}}{\left|\Delta F_{n}\right|} \lambda_{n},
$$

where $\lambda_{n}$ represents the step size.

Step 6: Let $\mathrm{n}=\mathrm{n}+1$, and repeat steps 2 to 5 as many times as is required to obtain a point near to the optimum point. As the process is repeated, the step sizes and $\delta \mathrm{x}_{\mathrm{n}}$ values are decreased by taking $\delta \mathrm{x}_{\mathrm{n}}=$ $\delta \mathrm{x}_{0} / \mathrm{n}$ and $\lambda_{\mathrm{n}}=\lambda_{0} / \mathrm{n}$ This is because as the central point approaches the desired region, maintaining those original values would lead to lower precision. Moreover, a maximum/minimum point is characterized by greater curvature and a firstorder model is only applicable to a very small region. 


\subsection{Ordinal RSM}

While cardinal values have traditionally been used for the calculation of the gradient, the ordinals of the responses are less subjective to the effect of noise. Hence, in the presence of noise, it is possible that the calculation of the gradient using ordinal will cause a faster convergence of the RSM.

Slight modifications have to be made to the algorithm for a typical RSM above for ordinal optimization:

Step 3: Instead of calculating the responses at the design points, the responses at the points are given ranks (i.e. $1,2,3, \ldots$ ).

Step 4: The gradient is calculated using the relative ranks instead of the actual responses. (i.e. Replace $\Delta F_{n}$ with $\Delta($ Rank $)$.)

\section{COMPARISON OF CARDINAL AND ORDINAL RSM}

We can assume that the performance of a system can be expressed as a function of its parameters, and hence predict the values for set parameters and hence carry out RSM on the system. The response is defined as the function value with the inclusion of noise. In an actual experiment, the function is not known, and performances have to be determined experimentally. In this case, as we are trying to compare cardinal and ordinal RSM in general, several functions are used so that a generalization can be formulated.

RSM is simulated on Microsoft Excel Spreadsheets. This allows the noise level to be adjusted and the values to be calculated easily and this is done electronically. The function can also be modified with minimal hassle.

Both cardinal and ordinal RSM are run for 4 different simple 2 dimensional mathematical functions:

$$
\begin{aligned}
& F_{1}(x, y)=(x-1)^{2}+(y+2)^{2}, \\
& F_{2}(x, y)=x+y-4 x^{2}-12 y^{2}-12 x y+10, \\
& F_{3}(x, y)=\left(y-\frac{5.1}{4 \pi^{2}} x^{2}+\frac{5}{\pi} x-6\right)^{2}+10\left(1-\frac{1}{8 \pi}\right) \cos x+10 \\
& F_{4}\left(x_{1}, x_{2}\right)=-\sum_{i=1}^{2} x_{i} \sin \left(\sqrt{\left|x_{i}\right|}\right),
\end{aligned}
$$

with different starting points, step sizes, $\delta x_{0}, \delta y_{0}$ values and noise levels. In each function, the number of steps needed to first reach a point in the vicinity of the optimum is tabulated. The mean number of steps taken by each form of RSM is found and compared to determine, for each function and noise level, which form of RSM requires fewer steps. The comparison is carried out in terms of consistency, accuracy (the distance from the optimum) and effi-

\begin{tabular}{|c|c|}
\hline Function & $\begin{array}{c}\text { Starting } \\
\text { points tested }\end{array}$ \\
\hline$F_{1}$ & \multirow{3}{*}{$\begin{array}{l}(0,0), \quad(5,5), \\
(-5,5),(5,-5), \\
(-5,-5)\end{array}$} \\
\hline$F_{2}$ & \\
\hline$F_{3}$ & \\
\hline$F_{4}$ & $\begin{array}{l}(425,425) \\
(450,450) \\
(400,450) \\
(450,400) \\
(400,400)\end{array}$ \\
\hline
\end{tabular}
ciency (the number of steps taken).

Table 1: Summary of Starting Points Tested for Each Function

The $\lambda_{0}, \delta x_{0}$, and $\delta y_{0}$ values tested are 5 and 10 .

For each particular case, a value of " noise" is added on to the function. The noise follows a normal cumulative distribution, with a mean of 0 and a standard deviation which is a percentage of $\lambda_{0}$. The chosen percentages are $0 \%, 20 \%, 50 \%$ and $80 \%$.

\section{RESULTS AND DISCUSSION}

The comparison of cardinal and ordinal RSM can be done in a few ways. The $90 \%$ confidence interval of each of these sets of values is calculated and used to assess the consistency of the RSM, as seen in the next section.

A graph can also be plotted of the distance between the $\left(x_{n}, y_{n}\right)$ values and the optimum with successive runs to demonstrate the convergence property of the RSM. The faster the points approach the optimum point, the steeper the gradient of the graph, and the better the convergence. This is used to test for the efficiency and accuracy of the RSM. However, the graph only shows the convergence for a particular condition, and cannot be used to evaluate the general trend for the function.

The response surface can also be mapped out using the software, Origin 7.5. This is used for further analysis of the function and the desired optimum region.

\section{$\underline{\text { Results }}$}

The following are the functions tested and the results of the RSM performed on them.

(1) $\begin{aligned} F_{1}(x, y) & =(x-1)^{2}+(y+2)^{2} \\ \min \left(F_{1}\right) & =F_{1}(1,-2)=0\end{aligned}$ 
Table 1: Summary of Results for $\mathrm{F}_{1}$

\begin{tabular}{|c|c|c|c|c|}
\hline \multicolumn{5}{|c|}{$\begin{array}{c}90 \% \text { Confidence Interval for number of steps to reach } \leq 1 \\
\text { unit away from optimum }\end{array}$} \\
\hline $\begin{array}{c}\text { Coefficient } \\
\text { of noise as } \\
\text { percentage } \\
\text { of step size }\end{array}$ & $0 \%$ & $20 \%$ & $50 \%$ & $80 \%$ \\
\hline $\begin{array}{c}\text { Cardinal } \\
\text { RSM }\end{array}$ & $4.1 \pm 0.5$ & $4.8 \pm 0.8$ & $8 \pm 3$ & $18 \pm 14$ \\
\hline $\begin{array}{c}\text { Ordinal } \\
\text { RSM }\end{array}$ & $4 \pm 1$ & $6 \pm 1$ & $15 \pm 10$ & $33 \pm 18$ \\
\hline
\end{tabular}

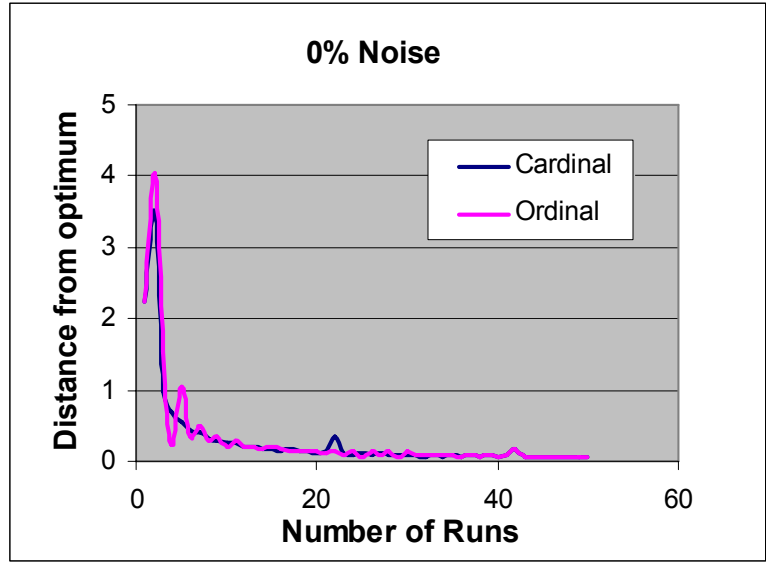

Figure 2: Convergence Graph for $F_{1}$ When Start Point $=$ $(0,0)$, Step Size, $\delta x, \delta y=5$, Noise $=0 \%$

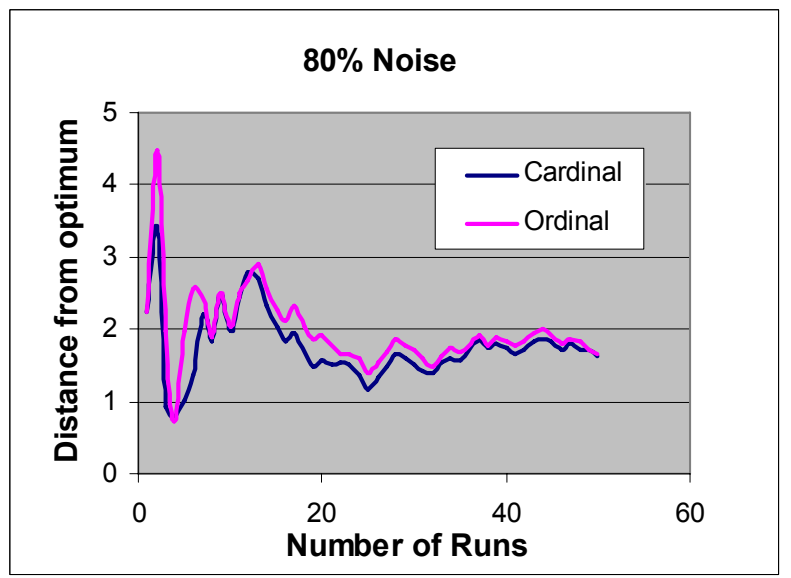

Figure 3: Convergence Graph for $F_{1}$ When Start Point $=$ $(0,0)$, Step Size, $\delta x, \delta y=5$, Noise $=80 \%$

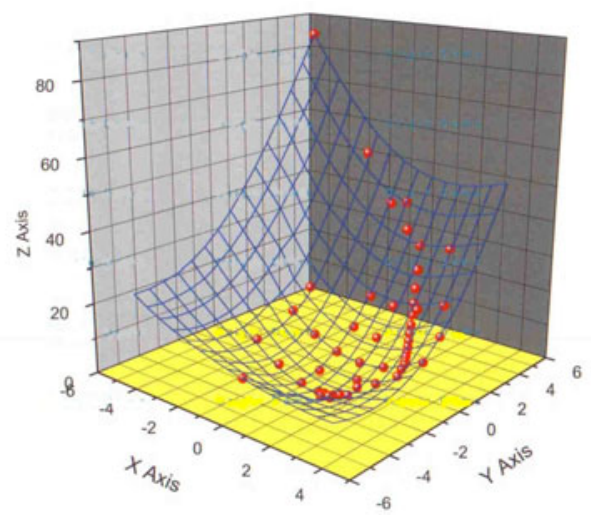

Figure 4: Surface Plot for $F_{1}$.

The two methods are comparable when the noise is low, reaching the optimum in about the same number of steps, though cardinal RSM seems to exhibit a more consistent convergence as step sizes and starting points are varied. As the degree of noise was increased up to a moderate level $(50 \%)$, it was seen that cardinal RSM emerged as the preferred choice.. When the noise is very large $(80 \%)$, the function does not seem to converge at all. This may be due to the fact that the step size decreases too quickly.

$$
\begin{aligned}
& F_{2}(x, y)=x+y-4 x^{2}-12 y^{2}-12 x y+10 \\
& \max \left(F_{2}\right)=F_{2}=(0.25,-0.084)=10.08
\end{aligned}
$$

Table 2: Summary of Results for $\mathrm{F}_{2}$

\begin{tabular}{|c|c|c|c|c|}
\hline \multicolumn{5}{|c|}{$90 \%$ Confidence Interval for number of steps to reach $\leq 1$} \\
unit away from optimum \\
\hline $\begin{array}{c}\text { Coefficient } \\
\text { of noise as } \\
\text { percentage } \\
\text { of step size }\end{array}$ & $0 \%$ & $20 \%$ & $50 \%$ & $80 \%$ \\
\hline $\begin{array}{c}\text { Cardinal } \\
\text { RSM }\end{array}$ & $10 \pm 2$ & $12 \pm 4$ & $14 \pm 6$ & $15 \pm 8$ \\
\hline $\begin{array}{c}\text { Ordinal } \\
\text { RSM }\end{array}$ & $8 \pm 2$ & $9 \pm 2$ & $12 \pm 4$ & $18 \pm 9$ \\
\hline
\end{tabular}




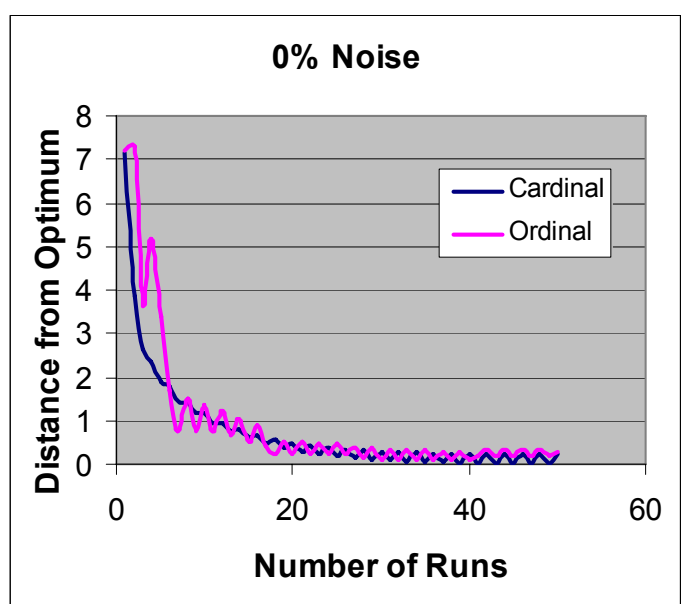

Figure 5: Convergence Graph for $F_{2}$ When Start Point $=$ $(-5,-5)$, Step Size, $\delta x, \delta y=10$, Noise $=0 \%$

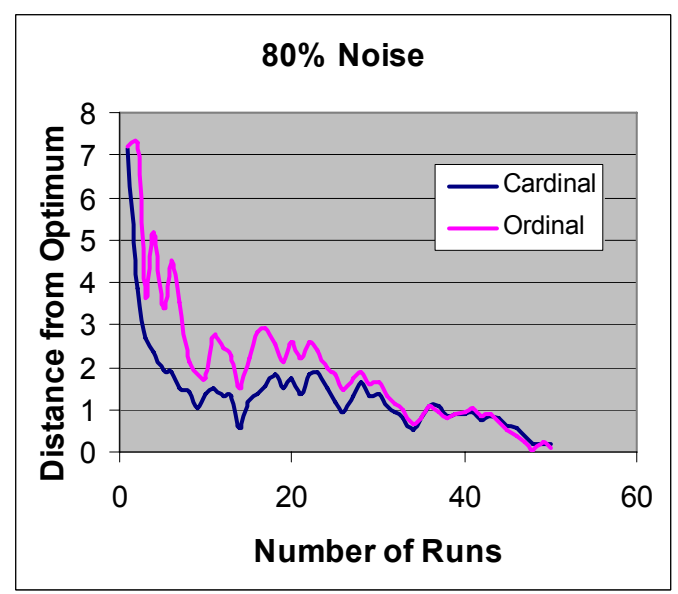

Figure 6: Convergence Graph for $F_{2}$ When Start Point $=$ $(-5,-5)$, Step Size, $\delta x, \delta y=10$, Noise $=80 \%$

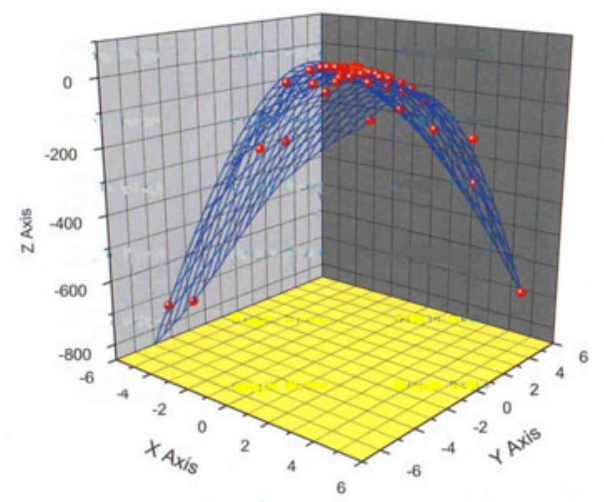

Figure 7: Surface Plot for $\mathrm{F}_{2}$.
On the whole, Table 2 shows that the ordinal RSM for $F_{2}$ performs better when the noise is small, but it worsens when the noise increases. This is because for ordinal RSM, there are only a few choices for the values of gradient. In general, although the orders of designs are more robust compared to the value of designs, a swap in ranks may cause a big change in gradient.

(3) Branin Function

$$
\begin{aligned}
& F_{3}(x, y)=\left(y-\frac{5.1}{4 \pi^{2}} x^{2}+\frac{5}{\pi} x-6\right)^{2}+10\left(1-\frac{1}{8 \pi}\right) \cos x+10 \\
& \min \left(F_{3}\right)=F_{3}(3.14,2.28)=F_{3}(9.43,2.42)=0.398
\end{aligned}
$$

Table 3: Summary of Results for $\mathrm{F}_{2}$

\begin{tabular}{|c|c|c|c|c|}
\hline \multicolumn{5}{|c|}{$90 \%$ Confidence Interval for number of steps to reach $\leq 1$} \\
unit away from optimum
\end{tabular}

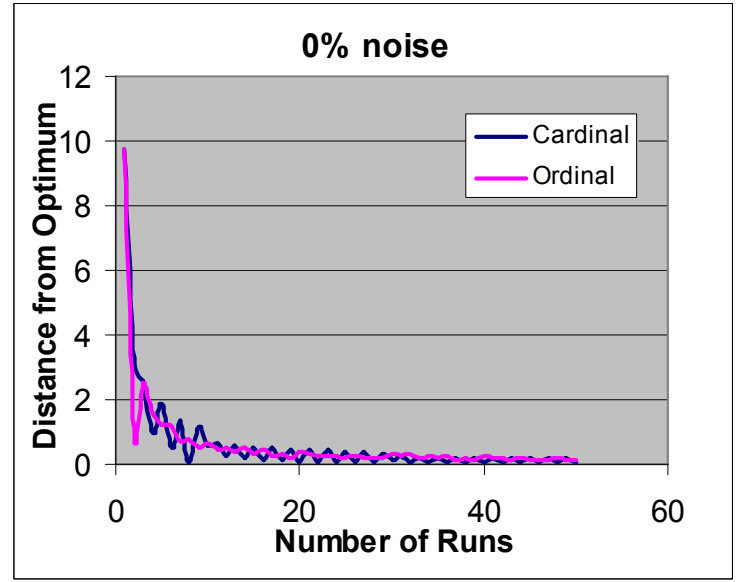

Figure 8: Convergence Graph for $F_{3}$ When Start Point $=$ $(0,0)$, Step Size $=10 \delta x, \delta y=5$, Noise $=0 \%$ 


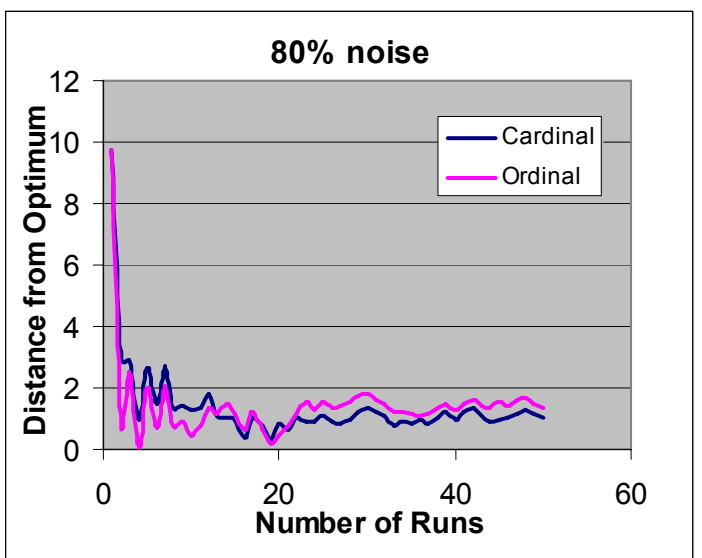

Figure 9: Convergence Graph for $F_{3}$ When Start Point $=$ $(0,0)$, Step Size $=10 \delta x, \delta y=5$, Noise $=80 \%$

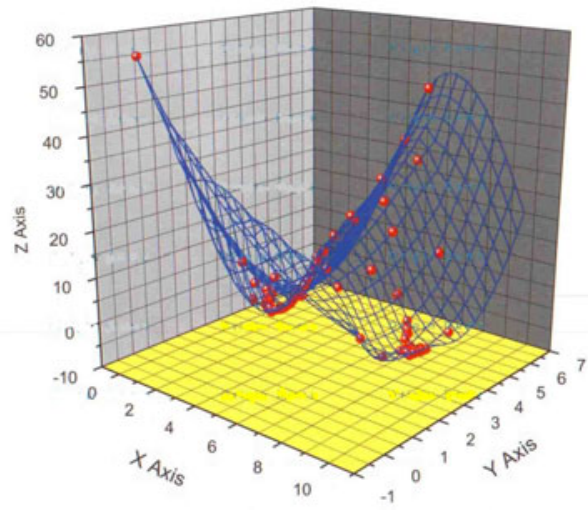

Figure 10: Surface Plot for $\mathrm{F}_{3}$.

This is a function which contains multiple peaks. When RSM was run, the different peaks were located depending on the starting point and the step size. Hence the number of steps for the RSM to obtain a point close to the optimum can be for any of the peaks, as they are of equal height. Likewise, the distance measured from the optimum is taken from the optimum that the RSM seems to be converging towards.

This case is unlike the previous two as the function exhibits good convergence property and the performance is not significantly affected by the presence of noise, regardless of the latter's extent. Both forms of RSM converge well and perform consistently. At all degrees of noise, ordinal RSM performs marginally better, but the differences in their performances are very small and hence can be deemed to be the same. This is because the magnitudes of the responses can get quite large at only a short distance from the minimum point (though not as large as those in (2)), such that randomness does not produce a marked change, in both the cardinal values and the ranks.
(4) Schwefel's problem:

$$
\begin{aligned}
& F_{4}\left(x_{1}, x_{2}\right)=-\sum_{i=1}^{2} x_{i} \sin \left(\sqrt{\left|x_{i}\right|}\right) \\
& \min \left(F_{4}\right)=F_{4}(421, \cdots, 421)=-838
\end{aligned}
$$

Table 4: Summary of Results for $\mathrm{F}_{4}$

\begin{tabular}{|c|c|c|c|c|}
\hline \multicolumn{5}{|c|}{$\begin{array}{c}90 \% \text { Confidence Interval for number of steps to reach } \leq 1 \\
\text { unit away from optimum }\end{array}$} \\
\hline $\begin{array}{c}\text { Coefficient } \\
\text { of noise as } \\
\text { percentage } \\
\text { of step size }\end{array}$ & $0 \%$ & $20 \%$ & $50 \%$ & $80 \%$ \\
\hline $\begin{array}{c}\text { Cardinal } \\
\text { RSM }\end{array}$ & $4.9 \pm 0.9$ & $7 \pm 2$ & $19 \pm 6$ & $23 \pm 7$ \\
\hline $\begin{array}{c}\text { Ordinal } \\
\text { RSM }\end{array}$ & $7 \pm 2$ & $17 \pm 2$ & $46 \pm 14$ & $79 \pm 12$ \\
\hline
\end{tabular}

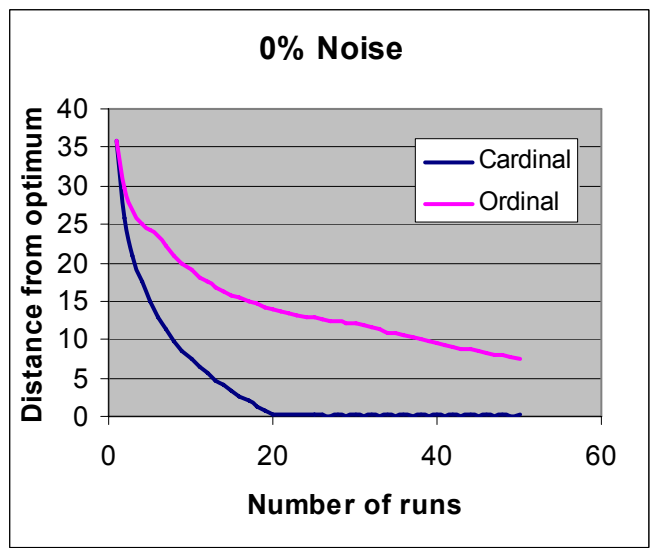

Figure 11: Convergence Graph for $F_{4}$ When Start Point $=$ $(400,450)$, Step Size, $\delta x, \delta y=10$, noise $=0 \%$

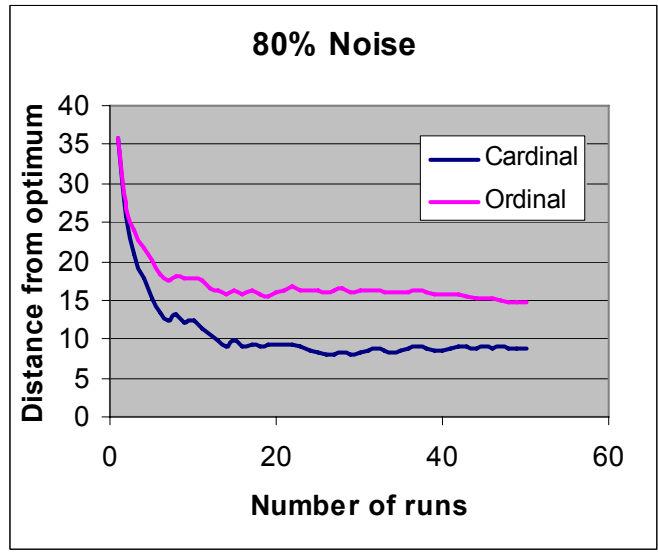

Figure 12: Convergence Graph for $F_{4}$ When Start Point $=$ $(400,450)$, Step Size, $\delta x, \delta y=10$, Noise $=80 \%$ 


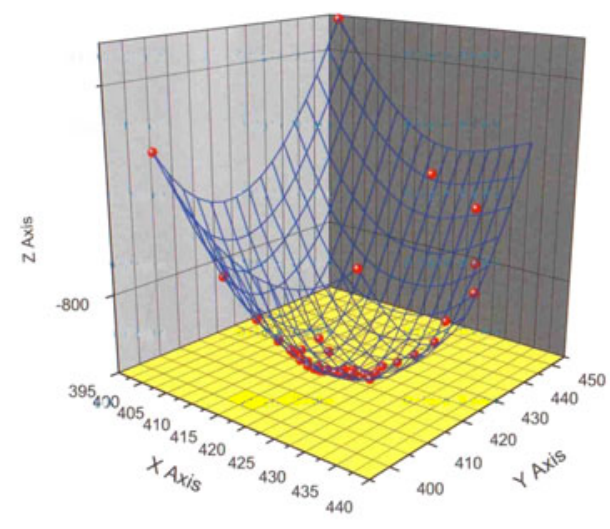

Figure 13: Surface Plot for $F_{4}$.

The performance of this function can be seen to be greatly affected by noise. In the presence of large noise, there are instances where the RSM never approaches the optimum value. In all cases, cardinal produces better results than ordinal RSM. This is because the function is a combination of algebraic and trigonometric parts, and trigonometric functions tend to display a large change for a small change in the variable. This is exacerbated by the multiplication of the algebraic part, such that the curvature of the surface is large, and neither method is effective in the presence of moderate or large noise. Cardinal RSM still has an edge over ordinal RSM as the gradient calculated using the exact values is a better estimation of the extent of the curvature as compared to that of ordinal RSM, which is more inaccurate due to the limited choice of gradients.

\section{CONCLUSION}

In many functions, cardinal RSM is still the preferred choice, especially where the response surface has a significant curvature over a small region. This is because while the responses can take on any values, the ranks are limited to $1,2,3,4,5$. Hence, the gradient calculated is not accurate. Also, in these experimental runs, ordinal RSM seems to work only when the noise is not large, hence differing from ordinal optimization, which is less affected by noise. We think that there is still room for improvement for ordinal RSM. An important possibility for future research would be to look at how to use the ordinal information to estimate the gradient.

The RSMs that we employed in this experiment were quite fundamental, and there were a lot of factors that we had not considered. Future work could be carried out to look at how these factors (e.g. choice of stopping rule and the step size chosen) will affect the performance of RSM. Since this is a largely empirical study aiming to explore the potential of ordinal RSM, there may be no general conclusion to make, which can only be reached after further study.

\section{REFERENCES}

C.M. Barnhart, J. E. Wieselthier, and A. Ephremides. 1994. Ordinal optimization by means of standard clock simulation and crude analytical models. Proc. $33^{\text {rd }}$ Conf. Decision and Control, Lake Buena Vista, FL, 2645-2647

Box, George E. P., and Norman R. Draper. 1987. Empirical Model Building and Response Surfaces. John Wiley and Sons, New York

C.G. Cassandras and G. Bao. 1994. A stochastic comparison algorithm for continuous optimization with estimations. Proc. $33^{\text {rd }}$ Conf. Decision and Control, Lake Buena Vista, FL

L.-Y. Dai. 1996. Convergence properties of ordinal comparison in the simulation of discrete event dynamic systems. J. Optimization Theory Appl., 91(2), 363-388

L.-Y. Dai and C.-H. Chen. 1997. Rate of convergence for ordinal comparison of dependent simulations in discrete event dynamic systems. J. Optimization Theory and Appl., 94 (1)

W.-B. Gong, Y.-C. Ho, and W. Zhai. 1992. Stochastic comparison algorithm for discrete optimization with estimations. Proc. $31^{\text {st }}$ IEEE Conf Decision and Control

Y.-C. Ho, R.S. Sreenivas, and P. Vakili. 1992. Ordinal Optimization in DEDS. J. Discrete Event Dynamic Syst., 3, 61-68

Y.-C. Ho and M. Deng. 1994. Large search space problems in ordinal optimization. Proc. Conf. Decision and Control

Y.-C Ho and M. E. Larson. 1995. Ordinal optimization approach to rare event probability problems. J. Discrete Event Dynamic Syst., 5, 281-301

Y.-C Ho, C.G. Cassandras, C.-H. Chen, and L.-Y. Dai. 2000. Ordinal Optimization and Simulation, Journal of Operational Research Society

L. H. Lee, T. W. E. Lau and Y. C. Ho. 1999. Explanation of goal softening in ordinal optimization. IEEE Transactions on Automatic Control, 44 (1), 94-99.

L. H. Lee, F. H. Abernathy, and Y. C. Ho. 2000. Production scheduling for apparel manufacturing systems. Production Planning and Control, 11 (3), 281-290.

D.C. Montgomery and G.C. Runger. 1994. Applied Statistics and Probability for Engineers, John Wiley and Sons, Inc, New York

Myers, Raymond H. and Douglas C. Montgomery. 2002. Response Surface Methodology: Process and Product Optimization Using Designed Experiments. $2^{\text {nd }}$ Edition, John Wiley and Sons, Inc., New York

Patsis, N. T., C. H. Chen, and M.E. Larson. 1997. SIMD Parallel Discrete Event Dynamic System Simulation. IEEE Trans. on Control Systems Technology, 5 (3), $30-41$ 
P. Vakili, L. Mollamustaflaglu, and Y.-C. Ho. 1992. Massively parallel simulation of a class of discrete event systems. Proc. IEEE Frontiers MPC Symp., Washington, DC

X. L. Xie. 1997. Dynamics and convergence rate of ordinal comparison of stochastic discrete event systems. IEEE Trans. Automat. Contr., 42, 586-590

\section{AUTHOR BIOGRAPHIES}

SARA JIAN OON is currently a student at Raffles Junior College, Singapore and has been admitted to Princeton University, Class of 2010. She will be pursuing a BSE program in Operations Research and Financial Engineering. This paper was written as part of the Science Research Programme organized by the Ministry of Education and the
National University of Singapore. Her email address is <sjoon6@gmail.com>.

LOO HAY LEE is an Associate Professor in the Department of Industrial and Systems Engineering, National University of Singapore. He received his B.S. (Electrical Engineering) degree from the National Taiwan University in 1992 and his S.M. and Ph.D. degrees in 1994 and 1997 from Harvard University. He is currently a senior member of IEEE, committee member of ORSS, and a member of INFORMS. His research interests include simulation-based optimization, production scheduling and sequencing and logistics and supply chain modeling. His email address is <iseleelh@nus.edu.sg> and his website is <www.ise.nus.edu.sg/staff/leelh/>. 